\title{
Solving Systems of Modular Equations in One Variable: How Many RSA-Encrypted Messages Does Eve Need to Know?*
}

\author{
Alexander May and Maike Ritzenhofen \\ Faculty of Mathematics \\ Ruhr-Universität Bochum, 44780 Bochum, Germany \\ alex.may@ruhr-uni-bochum.de, \\ maike.ritzenhofen@ruhr-uni-bochum.de
}

\begin{abstract}
We address the problem of polynomial time solving univariate modular equations with mutually co-prime moduli. For a given system of equations we determine up to which size the common roots can be calculated efficiently. We further determine the minimum number of equations which suffice for a recovery of all common roots. The result that we obtain is superior to Håstad's original RSA broadcast attack, even if Håstad's method is combined with the best known lattice technique due to Coppersmith. Namely, our reduction uses a slightly different transformation from polynomial systems to a single polynomial. Thus, our improvement is achieved by optimal polynomial modelling rather than improved lattice techniques. Moreover, we show by a counting argument that our results cannot be improved in general. A typical application for our algorithm is an improved attack on RSA with a smaller number of polynomially related messages.
\end{abstract}

Keywords: Chinese Remaindering, Coppersmith's method, Håstad's attack, systems of univariate modular polynomials.

\section{Introduction}

The RSA cryptosystem [14] is the public key cryptosystem which is most widely used in practice. Therefore, it has attracted the interest of many cryptanalysts since its invention in 1977 (compare e.g. [2]). In the following, let us denote by $N=p q$ the RSA modulus with prime factors $p$ and $q$, and let $\mathbb{Z}_{N}$ denote the ring of integers modulo $N$. Let $e$ be the public exponent, and let $d=e^{-1}$ $(\bmod \varphi(N))$ be the private key.

Attacks on RSA intend either to factorize the modulus and thereby recover the private key, or to compute $e$-th roots modulo $N$, i. e. to decrypt ciphertexts. The equivalence or inequivalence of these two problems is still open. However, partial results are known in restricted models [3410].

\footnotetext{
* This research was supported by the German Research Foundation (DFG) as part of the project MA 2536/3-1.
} 
In this paper we deal with the problem of extracting $e$-th roots. This is the well-known RSA problem: Given an RSA modulus $N$, a public exponent $e$ and a ciphertext $c \equiv m^{e}(\bmod N)$, find the corresponding plaintext $m$.

If $m^{e}<N$, the equation does not only hold in $\mathbb{Z}_{N}$ but over the integers, and we can calculate $m$ easily. This implies that encrypting small messages with small public exponents is insecure.

Let us look at the inhomogeneous case. Namely, suppose the most significant bits are known so that the unknown part remains small enough. Then we get the equation $(\tilde{m}+x)^{e} \equiv c(\bmod N)$, with $\tilde{m}$ denoting the known, $x$ the unknown part of the message. D. Coppersmith [6] showed that this inhomogeneous case can be solved efficiently under the same condition $x^{e}<N$.

Precisely, he showed that given a composite integer $N$ and a univariate polynomial $f(x) \in \mathbb{Z}_{N}[x]$ of degree $\delta$ one can determine all zeros smaller than $N^{\frac{1}{\delta}}$ efficiently. Hence, $(\tilde{m}+x)^{e} \equiv c(\bmod N)$ can be solved if $|x|<N^{\frac{1}{e}}$.

Now we may ask what happens if we get further information in form of additional polynomials? Can we then determine larger zeros as well?

There are two variants of systems of polynomial modular equations. Either there exist equations with the same modulus or all moduli are different. The first case was considered in Coppersmith, Franklin, Patarin and Reiter [7. They showed that it is usually sufficient to have two equations $f_{1}(x) \equiv 0(\bmod N)$ and $f_{2}(x) \equiv 0(\bmod N)$ in order to recover the common roots. Let $a$ be the common solution of the two equations. Then, $f_{1}(x)$ and $f_{2}(x)$ share a factor $(x-a)$. Computing the greatest common divisor $\operatorname{gcd}\left(f_{1}(x), f_{2}(x)\right)(\bmod N)$ reveals this factor if it is the only common factor. In the rare cases where the greatest common divisor is not linear, the method fails and further polynomials are needed. The running time of this method is $O\left(\delta \log ^{2} \delta\right)$ where $\delta$ is the degree of the given polynomials.

It is worth pointing out that a scenario with two RSA encryptions under coprime public exponents $\left(e_{1}, e_{2}\right)$ and a common modulus $N$ is a special case of this setting. Namely, an attacker has to find the common root $m$ of $f_{1}(x)=x^{e_{1}}-m^{e_{1}}$ $(\bmod N)$ and $f_{2}(x)=x^{e_{2}}-m^{e_{2}}(\bmod N)$. G. Simmons 16 has presented a neat attack for this special setting with running time polynomial in the bitlength of $\left(e_{1}, e_{2}\right)$. Namely, one computes integers $u_{1}, u_{2}$ such that $u_{1} e_{1}+u_{2} e_{2}=1$ with the help of the Extended Euclidean Algorithm. This gives us $m=\left(m^{e_{1}}\right)^{u_{1}}\left(m^{e_{2}}\right)^{u_{2}}$ $(\bmod N)$.

In this work, we focus on equations with different moduli $N_{1}, N_{2}, \ldots, N_{k} \in \mathbb{N}$. Without loss of generality, we assume that all moduli are composite as modular equations over finite fields can be solved efficiently (compare e.g. 11, Chapter 7.4). We further assume that the $N_{i}, i=1, \ldots, k$, are relatively prime. In case of our main application, RSA-moduli, we can otherwise compute prime factors of the $N_{i}$ by computing the greatest common divisor.

Before we define our polynomial roots problem in general, let us give a motivating cryptographic application. This application was introduced by J. Håstad in 8 ,9 and can be considered as an analogue of Simmon's attack in the setting of different RSA moduli. A user wishes to send the same message $m$ to several 
participants having different moduli and using plain RSA encryption without padding techniques. Suppose these users share the same public exponent $e=3$. Then, an attacker obtains three equations $m^{3} \equiv c_{i}\left(\bmod N_{i}\right)$ for $i=1,2,3$. He can make use of the fact that the $N_{i}$ are relatively prime and combine the equations by the Chinese Remainder Theorem. Thus, he gets $m^{3}\left(\bmod N_{1} N_{2} N_{3}\right)$ and is able to determine $m$ in $\mathbb{Z}$ as $m^{3}<N_{1} N_{2} N_{3}$. Therefore, the attacker solves the system of polynomial equations $f_{i}(x) \equiv x^{3}-c_{i} \equiv 0\left(\bmod N_{i}\right), i=1,2,3$, with the common root $m$.

Now let us generalize to arbitrary polynomial equations. We define the problem of solving systems of modular univariate polynomial equations (SMUPEproblem).

Definition 1 (SMUPE-problem). Let $k \in \mathbb{N}, \delta_{1}, \ldots, \delta_{k} \in \mathbb{N}$, and let $N_{1}, \ldots$, $N_{k} \in \mathbb{N}$ be mutually co-prime composite numbers of unknown factorization. Suppose $N_{1}<N_{2}<\ldots<N_{k}$. Let $f_{1}(x), \ldots, f_{k}(x)$ be polynomials of degree $\delta_{1}, \ldots, \delta_{k}$ in $\mathbb{Z}_{N_{1}}[x], \ldots, \mathbb{Z}_{N_{k}}[x]$, respectively. Let

$$
\begin{aligned}
f_{1}(x) & \equiv 0 \quad\left(\bmod N_{1}\right) \\
f_{2}(x) & \equiv 0 \quad\left(\bmod N_{2}\right) \\
& \vdots \\
f_{k}(x) & \equiv 0 \quad\left(\bmod N_{k}\right)
\end{aligned}
$$

be a system of univariate polynomial equations.

Let $X \leq N_{1}, X \in \mathbb{R}$. Find all common roots $x_{0}$ of (11) with size $\left|x_{0}\right|<X$.

Our goal is to compute an upper bound $X$ for which the SMUPE-problem is solvable in time polynomial in $\prod_{i=1}^{k} \delta_{i}$ and in the bitlength of $\prod_{i=1}^{k} N_{i}$. This upper bound will give us a condition on the number of equations $k$ in terms of $\delta_{i}$ and $N_{i}$. This will enable us to compute the minimal $k$ such that the SMUPEproblem can be computed up to the bound $X=N_{1}$, i.e. system (11) can be solved efficiently.

J. Håstad [9] gave the following algorithm for solving the SMUPE-problem. Let $\delta \in \mathbb{N}$ be the maximum degree of all polynomials occuring in the system, i. e. $\delta:=\max _{i=1, \ldots, k}\left\{\delta_{i}\right\}$. One first multiplies the given polynomials with $x^{\delta-\delta_{i}}$ to adjust their degrees. Then one combines the resulting polynomials using the Chinese Reminder Theorem to a univariate polynomial $f(x)$ with the same roots modulo $\prod_{i=1}^{k} N_{i}$. Applying lattice reduction methods, J. Håstad derived $k>\frac{\delta(\delta+1)}{2}$ as a lower bound on the number of polynomials for efficiently finding all roots $x_{0}$ with $\left|x_{0}\right|<N_{1}$. This bound can be easily improved to $k \geq \delta$ by directly applying Coppersmith's lattice techniques [6] to $f(x)$ (see e.g. [2]).

Our contribution: We give a different construction to combine all $k$ polynomial equations into a single equation $f(x) \equiv 0\left(\bmod \prod_{i=1}^{k} N_{i}\right)$. Instead of multiplying the polynomials by powers of $x$ like in Håstad's approach, we take powers of the polynomials $f_{i}(x)$ themselves. This results in the condition $\sum_{i=1}^{k} \frac{1}{\delta_{i}} \geq 1$ for 
solving the SMUPE-problem for all $x_{0}$ with $\left|x_{0}\right|<N_{1}$. In case all polynomials share the same degree $\delta$ this corresponds to Håstad's condition $k \geq \delta$. For polynomials of different degrees, however, our new condition is superior. Especially, a few polynomials of low degree suffice.

The paper is organized as follows. In Section 2, we review Coppersmith's result from [6] and the Chinese Remainder Theorem for polynomials. In Section 3, we prove the new sufficient condition on the number of polynomials that is needed to recover all common roots efficiently. The improved RSA broadcast attack is given as an application in Section 4. In Section 5, we show that our condition cannot be improved in general by giving an example for which the condition is optimal.

\section{Preliminaries}

The problem of solving modular univariate polynomial equations is believed to be difficult in general. Under some restrictions on the roots however, this is not the case. In [6], D. Coppersmith showed how to provably determine zeros of modular univariate equations with sufficiently small size.

Theorem 1 (Coppersmith [6]). Let $f(x)$ be a monic polynomial of degree $\delta \in \mathbb{N}$ in one variable modulo an integer $N$ of unknown factorization. Let $X$ be a bound on the desired solution $x_{0}$. If $X \leq N^{\frac{1}{\delta}}$ then we can find all integers $x_{0}$ such that $f\left(x_{0}\right) \equiv 0(\bmod N)$ and $\left|x_{0}\right| \leq X$ in time $O\left(\delta^{5}(\delta+\log N) \log N\right)$.

The running time can be achieved by using an algorithm of Nguyen, Stehlé [13] for the LLL lattice basis reduction step (see [1112]).

The SMUPE-problem can be reduced to the problem of solving a single univariate polynomial equation by combining the equations into a single one with the same solutions. Then we can apply Theorem 11. A possible way to combine equations is by Chinese Remaindering which is described e.g. in 915.

Theorem 2 (Chinese Remainder Theorem). Let $k \in \mathbb{Z}$. Let $\delta \in \mathbb{N}, \delta>1$. For $i=1, \ldots, k$ let $N_{i} \in \mathbb{N}$ be pairwise relatively prime numbers, and let $f_{i}(x) \in$ $\mathbb{Z}[x]$ be polynomials of degree $\delta$.

Then there exists a unique polynomial $f(x)$ modulo $M:=\prod_{i=1}^{k} N_{i}$ such that

$$
f(x) \equiv f_{i}(x) \quad\left(\bmod N_{i}\right)
$$

The polynomial $f(x)$ can be determined in time $O\left(\delta \log ^{2} M\right)$.

Proof. Let $M:=\prod_{i=1}^{k} N_{i}, M_{i}:=\frac{M}{N_{i}}$ and $M_{i}^{\prime}$ be the inverse of $M_{i}$ modulo $N_{i}$ for $i=1, \ldots, k$. The existence of such an inverse is guaranteed by $\operatorname{gcd}\left(M_{i}, N_{i}\right)=1$. Then

$$
f(x)=\sum_{i=1}^{k} M_{i} M_{i}^{\prime} f_{i}(x)
$$


is the desired solution. If we look at $f(x)$ modulo $N_{j}$ for $j \in\{1, \ldots, k\}$, all summands with index $i \neq j$ cancel out (as $N_{j}$ divides $M_{i}$ ) and $M_{j} M_{j}^{\prime} f_{j}(x) \equiv$ $f_{j}(x)\left(\bmod N_{j}\right)$.

Now suppose that $g(x)$ is another solution fulfilling the required conditions. Then, $f(x)-g(x) \equiv 0\left(\bmod N_{i}\right)$ for all $i=1, \ldots, k$, and therefore also $f(x) \equiv$ $g(x)(\bmod M)$.

Multiplication modulo $M$ and calculating the inverses by the Extended Euclidean Algorithm can be performed in time $O\left(\log ^{2} M\right)$. Determining all coefficients of $f$ then gives us $O\left(\delta \log ^{2} M\right)$ for the complete algorithm.

\section{An Improved Algorithm for Solving SMUPE}

For notational convenience let us briefly recall the SMUPE-problem. Given $k \in \mathbb{N}, N_{1}, \ldots, N_{k} \in \mathbb{N}$, mutually co-prime composite numbers of unknown factorization, such that $N_{1}<\ldots<N_{k}$, and a system of polynomial equations

$$
\begin{aligned}
f_{1}(x) & \equiv 0 \quad\left(\bmod N_{1}\right) \\
f_{2}(x) & \equiv 0 \quad\left(\bmod N_{2}\right) \\
& \vdots \\
f_{k}(x) & \equiv 0 \quad\left(\bmod N_{k}\right),
\end{aligned}
$$

where $f_{1}(x), \ldots, f_{k}(x)$ are of degree $\delta_{1}, \ldots, \delta_{k} \in \mathbb{N}$ in $\mathbb{Z}_{N_{1}}[x], \ldots, \mathbb{Z}_{N_{k}}[x]$, respectively.

Let $X \leq N_{1}, X \in \mathbb{R}$. Recover all solutions $x_{0}$ of (1) with $\left|x_{0}\right|<X$.

Considering for example Coppersmith's method (Theorem 10) for the first equation in (11), only small roots $x_{0}$ with $\left|x_{0}\right|<N_{1}^{\frac{1}{\delta_{1}}}$ can be found in polynomial time. By considering further equations this bound can be improved until all solutions can be found eventually.

By Håstad's algorithm in combination with Theorem 1 the condition $k \geq \delta$ with $\delta:=\max _{i=1, \ldots, k}\left\{\delta_{i}\right\}$ is sufficient to solve a system of equations efficiently. However, this condition is clearly not optimal as the following trivial example shows. Let $N_{1}<\ldots<N_{4}$ and take the following equations.

$$
\begin{array}{ll}
x^{3} \equiv c_{1} & \left(\bmod N_{1}\right) \\
x^{3} \equiv c_{2} & \left(\bmod N_{2}\right) \\
x^{3} \equiv c_{3} & \left(\bmod N_{3}\right) \\
x^{5} \equiv c_{4} & \left(\bmod N_{4}\right)
\end{array}
$$

Then $k=4<5=\delta$, i.e. the condition is not fulfilled. However, if we just take the first three equations, we are able to compute all common solutions smaller than $N_{1}$. This gives us the intuition that the proportion of higher and lower degrees of the polynomials ought to be taken into account. Let us now change 
the given example a little bit into a non-trivial one, so that no subsystem of the equations fulfills the sufficient condition.

$$
\begin{aligned}
& x^{3} \equiv c_{1} \quad\left(\bmod N_{1}\right) \\
& x^{3} \equiv c_{2} \quad\left(\bmod N_{2}\right) \\
& x^{5} \equiv c_{3} \quad\left(\bmod N_{3}\right) \\
& x^{5} \equiv c_{4} \quad\left(\bmod N_{4}\right)
\end{aligned}
$$

The parameters $k$ and $\delta$ and the $N_{i}$ remain the same. Can we still determine all solutions? We notice that we can transform the first equation by squaring into

$$
x^{6} \equiv 2 c_{1} x^{3}-c_{1}^{2} \quad\left(\bmod N_{1}^{2}\right)
$$

Applying Theorem 1 to this equation, we can find all solutions $x$ for which $|x|<\left(N_{1}^{2}\right)^{\frac{1}{6}}=N_{1}^{\frac{1}{3}}$ holds. This is the same bound which we get for the roots of the original equation $x^{3} \equiv c_{1}\left(\bmod N_{1}\right)$. We proceed with the second equation in the same way, then multiply the two other equations by $x$ and finally combine all the equations by Theorem 2 (Chinese Remainder Theorem). This gives us

$$
x^{6} \equiv a_{1}\left(2 c_{1} x^{3}-c_{1}^{2}\right)+a_{2}\left(2 c_{2} x^{3}-c_{2}^{2}\right)+a_{3} x c_{3}+a_{4} x c_{4} \quad\left(\bmod N_{1}^{2} N_{2}^{2} N_{3} N_{4}\right),
$$

where the $a_{i}$ are the coefficients from the Chinese Remainder Theorem, i.e. $a_{i} \equiv 1\left(\bmod N_{i}\right), a_{i} \equiv 0\left(\bmod N_{j}\right), j \neq i$. The above equation can be solved in $\mathbb{Z}$ for $x$ with $|x|<\left(N_{1}^{2} N_{2}^{2} N_{3} N_{4}\right)^{\frac{1}{6}}$. This condition is fulfilled for any $x$ with $|x|<N_{1}=\left(N_{1}^{6}\right)^{\frac{1}{6}} \leq\left(N_{1}^{2} N_{2}^{2} N_{3} N_{4}\right)^{\frac{1}{6}}$. Therefore, we can determine all solutions of the above system of equations, although the condition $k \geq \delta$ is not fulfilled.

In order to generalize our approach we make the following crucial observation. Let $f(x)$ be a polynomial of degree $\delta$. Let $f(x) \equiv 0(\bmod N)$ for $N \in \mathbb{N}$, and let $m \in \mathbb{N}$. Then $g(x):=f^{m}(x) \equiv 0\left(\bmod N^{m}\right)$. The solutions $x$ with $|x|<N$ of the two equations remain unchanged. Moreover, with Coppersmith's Theorem 1 we can determine those solutions for which the condition $|x|<N^{\frac{1}{\delta}} \Leftrightarrow|x|<$ $\left(N^{m}\right)^{\frac{1}{m \delta}}$ holds. Thus, Coppersmith's bound is invariant under taking powers of the polynomial $f(x)$.

As opposed to our approach, in Håstad's algorithm one does not take powers of the polynomials but multiplications of polynomials with powers of $x$. This increases the degree of the polynomial but leaves the modulus unchanged. Let $f(x)$ be a polynomial of degree $\delta$ with $f(x) \equiv 0(\bmod N)$ for $N \in \mathbb{N}$. Then with $\gamma>\delta$ the equation $g(x):=x^{\gamma-\delta} f(x) \equiv 0(\bmod N)$ contains all the solutions $x$ of $f(x)$ with $|x|<N$. However, applying Coppersmith's method to determine roots of $g(x)$ we only get roots $x$ with $|x|<N^{\frac{1}{\gamma}}<N^{\frac{1}{\delta}}$. So obviously, Coppersmith's bound is not invariant under multiplication with powers of $x$. This explains why we obtain a superior bound on the size of the roots.

In the following analysis we will restrict ourselves to monic polynomials. If one of the given polynomials $f_{i}(x)$ is not monic, either the coefficient of the leading monomial is invertible, or we can find a factor of the modulus. In the first case, we make the polynomial monic by multiplication with the inverse of 
the leading coefficient. In the latter case, we obtain for RSA moduli the complete factorization, which in turn allows for efficiently solving this polynomial equation modulo the prime factors.

Theorem 3. Let $\left(f_{i}, \delta_{i}, N_{i}\right), i=1, \ldots, k$, be an instance of the SMUPE-problem with monic $f_{i}$. Define $M:=\prod_{i=1}^{k} N_{i}^{\frac{\delta}{\delta_{i}}}$ with $\delta:=\operatorname{lcm}\left\{\delta_{i}, i=1, \ldots, k\right\}$. Then the SMUPE-problem can be solved for all $x_{0}$ with

$$
\left|x_{0}\right|<M^{\frac{1}{\delta}}
$$

in time $O\left(\delta^{6} \log ^{2} M\right)$.

Proof. Let $x_{0}$ be a solution of the system of polynomial equations (11). Then $x_{0}$ is a solution of

$$
f_{i}^{\frac{\delta}{\delta_{i}}}(x) \equiv 0 \quad\left(\bmod N_{i}^{\frac{\delta}{\delta_{i}}}\right) \text { for all } i=1, \ldots, k .
$$

All these equations have common degree $\delta$ and are monic.

Combining them by Chinese Remaindering yields a polynomial $f(x)$ of degree $\delta$ such that $x_{0}$ is a solution of $f(x) \equiv 0(\bmod M)$ with $M:=\prod_{i=1}^{k} N_{i}^{\frac{\delta}{\delta_{i}}}$. Moreover, this polynomial is still monic.

For the coefficient $a_{\delta}$ of the monomial $x^{\delta}$ in $f(x)$ it holds that $a_{\delta} \equiv 1$ $\left(\bmod N_{i}^{\frac{\delta}{\delta_{i}}}\right)$ for all $i=1, \ldots, k$ and therefore $a_{\delta} \equiv 1(\bmod M)$.

The above step can be performed in time $O\left(\delta \log ^{2} M\right)$ by Theorem 2 , With Theorem 1 all solutions $x_{0}$ of the above equation which fulfill $\left|x_{0}\right| \leq M^{\frac{1}{\delta}}=$ $\left(\prod_{i=1}^{k} N_{i}^{\frac{\delta}{\delta_{i}}}\right)^{\frac{1}{\delta}}$ can be found in time $O\left(\delta^{5}(\delta+\log M) \log M\right)$. The result can therefore be obtained in time $O\left(\delta^{6} \log ^{2} M\right)$.

Remark 1. The same result is obtained by applying Coppersmith's method [6] directly to the polynomials $f_{1}(x), \ldots, f_{k}(x)$ instead of $f(x)$.

Theorem 3 immediately gives us a sufficient condition on $k$ and the $\delta_{i}$ for solving the SMUPE-problem for all $x_{0} \in \mathbb{Z}_{N_{1}}$.

Corollary 1. The SMUPE-problem can be solved for all $x_{0} \in \mathbb{Z}_{N_{1}}$ in time $O\left(\delta^{6} \log ^{2} M\right)$ provided that

$$
\sum_{i=1}^{k} \frac{1}{\delta_{i}} \geq 1 .
$$

Proof. Let $x_{0}$ be a common solution to all the equations. An application of Theorem 3 gives us $\left|x_{0}\right|<M^{\frac{1}{\delta}}:=\left(\prod_{i=1}^{k} N_{i}^{\frac{\delta}{\delta_{i}}}\right)^{\frac{1}{\delta}}$ as an upper bound for all roots that can be computed in time $O\left(\delta^{6} \log ^{2} M\right)$. As $\left(\prod_{i=1}^{k} N_{i}^{\frac{\delta}{\delta_{i}}}\right)^{\frac{1}{\delta}} \geq \prod_{i=1}^{k} N_{1}^{\frac{1}{\delta_{i}}}=$ $N_{1}^{\sum_{i=1}^{k} \frac{1}{\delta_{i}}} \geq N_{1}$ all solutions $x_{0} \in \mathbb{Z}_{N_{1}}$ can be found.

This gives us an algorithm to solve the SMUPE-problem with running time polynomial in the bitsize of the $N_{i}, i=1, \ldots, k$, if $\delta$ is polynomial in the bitsize of the $N_{i}$. 
Comparing this to the result due to Håstad and Coppersmith we observe that in the case $\delta:=\delta_{1}=\ldots=\delta_{k}$ the sufficient condition is $k \geq \delta$ with both methods. For different $\delta_{i}$ however, our method is always superior. Taking e.g. the illustrating example with public exponents $(3,3,5,5)$ from the beginning of this section, we see that our new condition $\frac{1}{3}+\frac{1}{3}+\frac{1}{5}+\frac{1}{5}=\frac{16}{15} \geq 1$ is fulfilled.

\section{Application: RSA with Polynomially Related Messages}

A typical example in which polynomially related messages occur is an RSA broadcast scenario. Assume a user wants to broadcast a message $m$ to $k$ different users using an RSA encryption scheme with public exponents $e_{1}, \ldots, e_{k}$ and coprime public moduli $N_{1}<\ldots<N_{k}$. From the ciphertexts $c_{1}\left(\bmod N_{1}\right), \ldots, c_{k}$ $\left(\bmod N_{k}\right)$ an attacker can compute the message $m$ if $m$ is smaller than the upper bound given in Theorem 3 . He sets $f_{i}(x)=x^{e_{i}}-c_{i}\left(\bmod N_{i}\right)$ and applies Theorem 3 ,

In order to avoid sending various encryptions of the same message, a user might add some randomness $r_{i}$ and then encrypt the linearly related messages $\left(m+r_{i}\right), i=1, \ldots, k$, instead of $m$. However, if the attacker gets to know the randomness, he can calculate $F_{i}(x):=f_{i}\left(x+r_{i}\right)\left(\bmod N_{i}\right)$ and analyze the system of equations $F_{i}(x) \equiv 0\left(\bmod N_{i}\right), i=1, \ldots, k$. As degree, modulus and leading coefficient are the same for $F_{i}(x)$ and $f_{i}(x)$, the upper bound on $m$, up to which $m$ can be recovered efficiently, also remains unchanged. More generally, taking polynomially related messages instead of linearly related ones, the degree of $F_{i}(x), i=1, \ldots, k$, changes from $e_{i}$ to $e_{i} \gamma_{i}$, where $\gamma_{i}$ is the degree of the known polynomial relation.

Theorem 4. Let $k \in \mathbb{N},\left(e_{i}, N_{i}\right), i=1, \ldots, k$, be $R S A$ public keys with $N_{1}<$ $N_{2}<\ldots<N_{k}$ and co-prime $N_{i}$. Furthermore, let $m \in \mathbb{Z}_{N_{1}}$ and let $g_{i}(x) \in \mathbb{Z}[x]$ be polynomials of degree $\gamma_{i} \in \mathbb{N}$ with $a_{i \gamma_{i}}$ the coefficient of $x^{\gamma_{i}}$ for $i=1, \ldots, k$. Let $c_{1}, \ldots, c_{k}$ be the RSA-encryptions of $g_{i}(m)$ under the public key $\left(e_{i}, N_{i}\right)$. Define $\delta_{i}:=e_{i} \gamma_{i}$ and $M:=\prod_{i=1}^{k} N_{i}^{\frac{\delta}{\delta_{i}}}$ with $\delta:=\operatorname{lcm}\left\{\delta_{i}, i=1, \ldots, k\right\}$.

Then an adversary can recover the message $m$ in time $O\left(\delta^{6} \log ^{2} M\right)$ provided that

$$
\sum_{i=1}^{k} \frac{1}{\delta_{i}} \geq 1 .
$$

Proof. Without loss of generality we assume that all $a_{i \gamma_{i}}$ are invertible modulo $N_{i}$. (Otherwise $\operatorname{gcd}\left(a_{i \gamma_{i}}, N_{i}\right)$ and $\frac{N_{i}}{\operatorname{gcd}\left(a_{i \gamma_{i}}, N_{i}\right)}$ will give us the factorization of $N_{i}$ for at least one $i \in\{1, \ldots, k\}$. We can then compute $m$ modulo the prime factors. This can be done efficiently (see [1])).

We are looking for a solution $m$ of $f_{i}(x):=g_{i}(x)^{e_{i}}-c_{i} \equiv 0\left(\bmod N_{i}\right), i=$ $1, \ldots, k$. However, the polynomials $f_{i}(x)$ are not necessarily monic. Therefore, we modify them slightly to be able to apply Corollary 1 Let $F_{i}(x):=a_{i \gamma_{i}}^{-e_{i}}\left(g_{i}(x)^{e_{i}}-\right.$ $\left.c_{i}\right)\left(\bmod N_{i}\right), i=1, \ldots, k$. Hence, $F_{i}(x)$ is a monic polynomial of degree $\delta_{i}=$ $e_{i} \gamma_{i}$. The theorem then directly follows as an application of Corollary 1 . 


\section{Optimality of Our Bound for Solving SMUPE}

In this section, we will see that the condition $\left|x_{0}\right|<M^{\frac{1}{\delta}}$ for efficiently solving the SMUPE-problem given in Theorem 3 is optimal if the moduli $N_{i}$ are prime powers. This implies that the condition cannot be improved in general, unless we make use of the structure of the moduli or of the specific polynomials occuring in the system. Thus, our argument does not exclude the existence of superior conditions for special moduli, e.g. square-free $N_{i}$. Moreover, our formula captures the intuition that equations of low degree $\delta_{i}$ comprise more information since they contribute to the sum in (3) with a larger term $\frac{1}{\delta_{i}}$ than equations with higher degree.

The counting argument that we use is a generalization of the argument in 5 to systems of polynomial equations instead of a single equation.

Let $k \in \mathbb{N}$. Let $p_{1}, \ldots, p_{k}$ be different prime numbers, $\delta_{1}, \ldots, \delta_{k} \in \mathbb{N}$ and $N_{1}:=p_{1}^{\delta_{1}}, \ldots, N_{k}:=p_{k}^{\delta_{k}}$. Suppose $N_{1}<\ldots<N_{k}$. Let us look at the following system of polynomial equations.

$$
\begin{aligned}
f_{1}(x):=x^{\delta_{1}} & \equiv 0 \quad\left(\bmod N_{1}\right) \\
f_{2}(x):=x^{\delta_{2}} \equiv 0 \quad\left(\bmod N_{2}\right) & \\
\vdots & \\
f_{k}(x):=x^{\delta_{k}} & \equiv 0 \quad\left(\bmod N_{k}\right)
\end{aligned}
$$

We would like to determine all solutions $x_{0}$ of this system with $\left|x_{0}\right|<N_{1}=p_{1}^{\delta_{1}}$. An application of Theorem 1 to a single equation $f_{i}(x) \equiv 0\left(\bmod N_{i}\right)$ efficiently yields all solutions $x_{0}$ with $\left|x_{0}\right|<\left(N_{i}\right)^{\frac{1}{\delta_{i}}}=p_{i}$. Furthermore, each multiple of $p_{i}$ is a solution of $f_{i}(x) \equiv 0\left(\bmod N_{i}\right)$. Thus, if $x_{0}$ is a multiple of $\prod_{i=1}^{k} p_{i}$, then $x_{0}$ is a common zero of all the polynomials.

Let $\delta:=\operatorname{lcm}\left\{\delta_{i}, i=1, \ldots, k\right\}$. We apply the same method as in the proof of Theorem 3 to the polynomial equations in system (44). Namely, we take their $\frac{\delta}{\delta_{i}}$ th powers and combine them by Chinese Remaindering (Theorem 2). This gives us an equation $f(x) \equiv x^{\delta}(\bmod M)$ with $M:=\prod_{i=1}^{k} N_{i}^{\frac{\delta}{\delta_{i}}}=\prod_{i=1}^{k} p_{i}^{\delta}$ with the same roots as in (41).

We assume that $M^{\frac{1}{\delta}}<N_{1}$. Otherwise $M^{\frac{1}{\delta}} \geq N_{1}>\left|x_{0}\right|$, i. e. the condition of Theorem 3 is fulfilled and there is nothing to be shown. Therefore, let $\epsilon>0$ such that $M^{\frac{1}{\delta}+\epsilon}<N_{1}$. Suppose now we could calculate all simultaneous solutions $x_{0}$ of the system such that $\left|x_{0}\right|<M^{\frac{1}{\delta}+\epsilon}=\left(\prod_{i=1}^{k} p_{i}\right)^{1+\delta \epsilon}$. Since we know that every integer multiple of $\prod_{i=1}^{k} p_{i}$ is a root of (4), the number of roots is roughly $2\left(\prod_{i=1}^{k} p_{i}\right)^{\delta \epsilon}$. This implies that we have exponentially many roots $x_{0}$ with $\left|x_{0}\right|<$ $M^{\frac{1}{\delta}+\epsilon}$, which we cannot even output in polynomial time. Consequently, there is no polynomial time algorithm that improves upon the exponent in the condition $\left|x_{0}\right|<M^{\frac{1}{\delta}}$ of Theorem 3 . 


\section{Acknowledgments}

We thank the anonymous reviewers of PKC 2008 for their helpful comments.

\section{References}

1. Bach, E., Shallit, J.: Algorithmic Number Theory, vol. 1, efficient algorithms. MIT Press, Cambridge (1996)

2. Boneh, D.: Twenty years of attacks on the RSA cryptosystem. Notices of the AMS (1999)

3. Boneh, D., Venkatesan, R.: Breaking RSA May Not Be Equivalent To Factoring. In: Nyberg, K. (ed.) EUROCRYPT 1998. LNCS, vol. 1403, pp. 59-71. Springer, Heidelberg (1998)

4. Brown, D.: Breaking RSA May Be As Difficult As Factoring., Cryptology ePrint Archive Report 2005/380 (2005)

5. Coppersmith, D.: Finding Small Solutions to Small Degree Polynomials. In: Silverman, J.H. (ed.) CaLC 2001. LNCS, vol. 2146, pp. 20-31. Springer, Heidelberg (2001)

6. Coppersmith, D.: Small solutions to polynomial equations and low exponent vulnerabilities. Journal of Cryptology 10(4), 223-260 (1997)

7. Coppersmith, D., Franklin, M., Patarin, J., Reiter, M.: Low-exponent RSA with related messages. In: Maurer, U.M. (ed.) EUROCRYPT 1996. LNCS, vol. 1070, pp. 1-9. Springer, Heidelberg (1996)

8. Håstad, J.: On Using RSA with Low Exponent in a Public Key Network. In: Williams, H.C. (ed.) CRYPTO 1985. LNCS, vol. 218, pp. 403-408. Springer, Heidelberg (1986)

9. Håstad, J.: Solving Simultaneous Modular Equations of Low Degree. SIAM Journal on Computing 17(2), 336-341 (1988)

10. Leander, G., Rupp, A.: On the Equivalence of RSA and Factoring Regarding Generic Ring Algorithms. In: Lai, X., Chen, K. (eds.) ASIACRYPT 2006. LNCS, vol. 4284, pp. 241-251. Springer, Heidelberg (2006)

11. Lenstra, A.K., Lenstra, H.W., Lovász, L.: Factoring polynomials with rational coefficients. Mathematische Annalen 261, 513-534 (1982)

12. May, A.: Using LLL-Reduction for Solving RSA and Factorization Problems: A Survey. LLL +25 Conference in honour of the 25 th birthday of the LLL algorithm (2007), http://www.cits.rub.de/personen/may.html

13. Nguyen, P.Q., Stehlé, D.: Floating Point LLL Revisited. In: Cramer, R.J.F. (ed.) EUROCRYPT 2005. LNCS, vol. 3494, pp. 215-233. Springer, Heidelberg (2005)

14. Rivest, R., Shamir, A., Adleman, L.: A Method for Obtaining Digital Signatures and Public-Key Cryptosystems. Communications of the ACM 21(2), 120-126 (1978)

15. Shoup, V.: A Computational Introduction to Number Theory and Algebra. Cambridge University Press, Cambridge (2005)

16. Simmons, G.: A "Weak" Privacy Protocol Using the RSA Crypto Algorithm. Cryptologia $7(2), 180-182(1983)$ 